\title{
Sub-barrier resonance fission and its effects on fission fragment properties, exemplified on ${ }^{234,238} U(n, f)$
}

\author{
A. Tudora ${ }^{1}$, F.-J. Hambsch ${ }^{2}$, and S. Oberstedt ${ }^{2}$ \\ ${ }^{1}$ University of Bucharest, Faculty of Physics, Bucharest-Magurele, RO-77125, Romania \\ ${ }^{2}$ EC-JRC-Institute for Reference Materials and Measurements (IRMM), 2440 Geel, Belgium
}

\begin{abstract}
The correlation between the sub-barrier resonant behaviour of fission crosssection of non-fissile actinides (pre-scission stage) and the visible fluctuations of their fission fragment and prompt neutron data (post-scission stage) around the incident energies of sub-barrier resonances is outlined and supported by quantitative results for two fissioning systems ${ }^{234,238} \mathrm{U}(\mathrm{n}, \mathrm{f})$. These quantitative results refer to both stages of the fission process: a) The pre-scission stage including the calculation of neutron induced cross-sections with focus on fission. Calculations are done in the frame of the refined statistical model for fission with sub-barrier effects also extended to take into account the multi-modal fission. b) The post-scission stage including the prompt neutron emission treated in the frame of the Point-by-Point model. Total quantities characterizing the fission fragments and the prompt neutrons obtained by averaging the Point-by-Point results as a function of fragment over the fission fragment distributions reveal variations around the energies of sub-barrier resonances in the fission crosssection.
\end{abstract}

\section{Properties of fission fragments and prompt neutrons at sub- threshold incident energies}

Since recent years fission fragment properties like mass and total kinetic energy (TKE) distributions are being measured systematically as a function of incident neutron energy (En). For instance in the case of non-fissile (fertile) actinides nearly complete emission yield data were measured for ${ }^{234,238} \mathrm{U}(\mathrm{n}, \mathrm{f})[1-3]$ in the En range where only the first fission chance is involved. A surprising discovery was that the characteristics of fission fragments (FF) show remarkable variation in the En range close to vibrational resonances in the sub-barrier fission cross-section. As an example we show in Figs. 1 and 2 the experimental average $<\mathrm{TKE}>$ as a function of En (lower parts) with the corresponding neutron induced fission cross-section (upper parts, existing experimental data plotted with different symbols and the present calculations with red solid line) [4]. The correlated behaviour of the fission cross-section and FF properties is best outlined in the cases of ${ }^{234,238} \mathrm{U}(\mathrm{n}, \mathrm{f})$ because these fertile nuclei benefit of experimental FF data measured at many En with a fine grid in the region of sub-barrier resonances in the fission cross-section.

This correlation makes the link between the two stages of the fission process (pre- and postscission) usually treated separately by two different classes of models. In the pre-scission stage only one single nucleus is involved, changing the shape on the fission path, starting from the ground state deformation, passing through different stages of deformation up to the rupture point. In this stage the 
neutron-induced fission is in competition with other reaction channels like elastic, inelastic and gamma-capture treated by the modelling of nuclear reaction mechanisms. The main quantity characterizing the pre-scission stage is the fission cross-section (obtained concomitantly with the neutron-induced cross-sections of competitive processes) as a function of En.

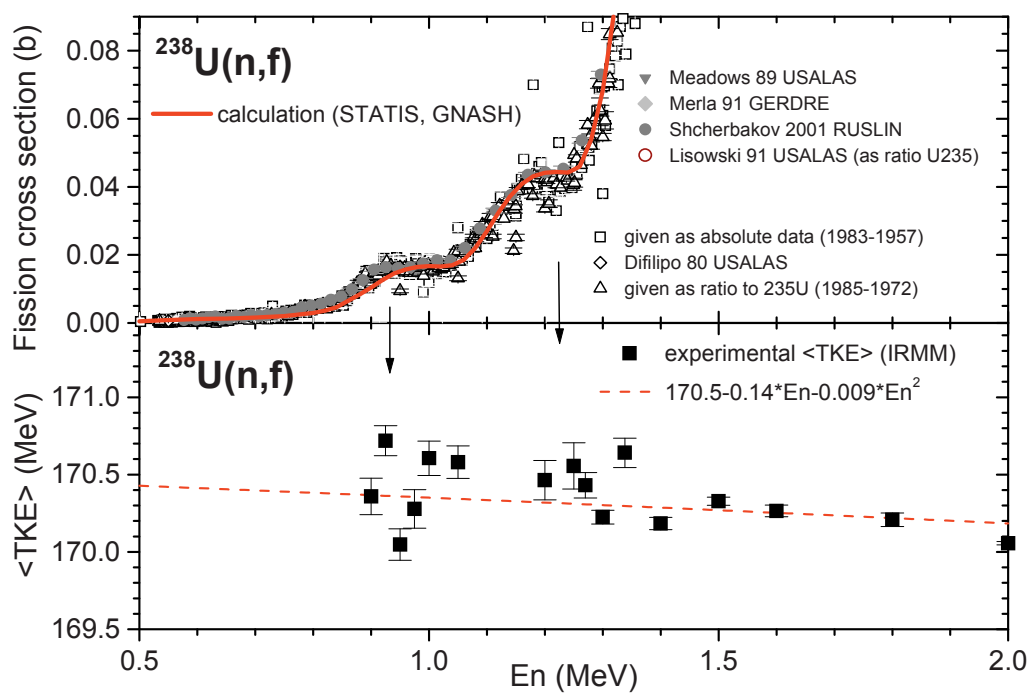

Fig.1. ${ }^{238} \mathrm{U}(\mathrm{n}, \mathrm{f})$ fission cross-section (upper part, experimental data with different symbols and present calculation with red solid line) and experimental $<\mathrm{TKE}>$ data (lower part with full squares) in the En range focusing the sub-barrier resonances.

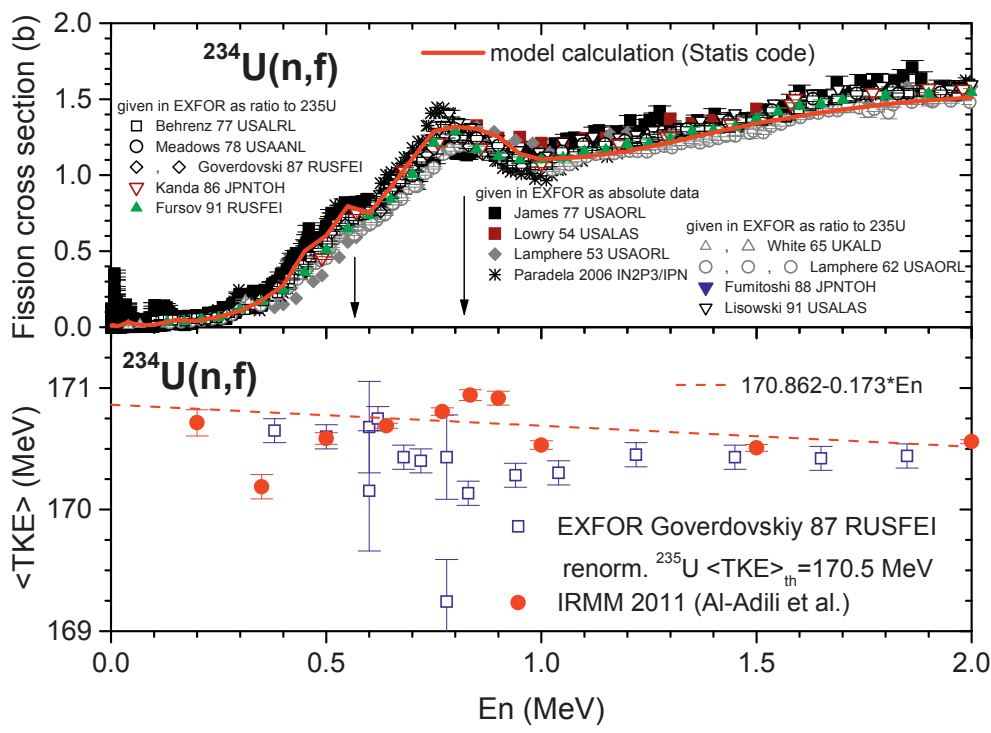

Fig.2. ${ }^{234} \mathrm{U}(\mathrm{n}, \mathrm{f})$ fission cross-section (upper part, experimental data with different symbols and present calculation with red solid line) and experimental $<\mathrm{TKE}>$ data (lower part, IRMM data with full circles and Goverdovskiy data with open squares) in the En range focusing the sub-barrier resonances.

In the post-scission stage many nuclei are involved (resulting from many possibilities of compound nucleus fragmentation), each FF emitting prompt neutrons and gammas according to its 
structure properties and excitation energy partition. This post-scission stage (called prompt fission) is characterized by quantities referring to both $\mathrm{FF}$ and emitted prompt neutrons and gammas. The FF quantities can be distributions (like $\mathrm{Y}(\mathrm{A}, \mathrm{TKE})$ ) or average quantities (like $<\mathrm{TKE}>$ ) as a function of En. The prompt neutron and gamma-ray quantities can be also given as a function of fragment (such as $v(\mathrm{~A}), \varepsilon(\mathrm{A}), \mathrm{E}_{\gamma}(\mathrm{A})$ and so on) or can be average quantities as a function of En (such as average prompt neutron multiplicity and spectra, average prompt gamma-ray energy and so on).

The correlation between the resonances in the fission cross-section (pre-scission stage involving one fissioning nucleus) and the quantities characterizing the prompt fission (the post scission stage involving many nuclei (the fission fragments), each of them emitting prompt neutrons and gammas) can be quantitatively connected in a consistent and coherent manner by taking into account the behaviour of average prompt fission quantities (obtained by averaging the quantities depending on fragments over the FF distributions).

In the frame of the multi-modal fission concept the coherence is assured by the behaviour of modal fission cross-sections as a function of En (pre-scission stage) that can be directly correlated with the behaviour (in the post-scission stage) of both modal prompt fission quantities as a function of En: the modal $\mathrm{FF}$ distributions $\mathrm{Y}_{\mathrm{m}}(\mathrm{A}), \mathrm{TKE}_{\mathrm{m}}(\mathrm{A})$ and the modal average quantities (for instance $<\mathrm{TKE}\rangle_{\mathrm{m}}$ ), the index " $\mathrm{m}$ " meaning the fission mode (usually S1, S2 and SL).

The sub-barrier resonances of the fission cross-section are reflected by an increase of the fission channel population (in the pre-scission stage) leading to an increase of FF distributions (in the postscission stage). This fact is illustrated by the fluctuations of average quantities characterizing the FF and the prompt neutron emission around the En of resonances. As examples we give in Fig. 3 the average heavy fragment mass $\left\langle\mathrm{A}_{\mathrm{H}}>\right.$ of ${ }^{238} \mathrm{U}(\mathrm{n}, \mathrm{f}$ ) (upper part) and the total average energy release $<$ Er $>$ of ${ }^{234} \mathrm{U}(\mathrm{n}, \mathrm{f})$ (lower part) as a function of En. $<\mathrm{Er}>$ is obtained by averaging the Q-values of fragment pairs over the FF mass and charge distributions. The Q-values of FF pairs and their charge distributions (taken as narrow Gaussians) do not change with En. Consequently the $<\mathrm{Er}>$ dependence on En (including the fluctuations around resonances) is given exclusively by $\mathrm{Y}(\mathrm{A})$.

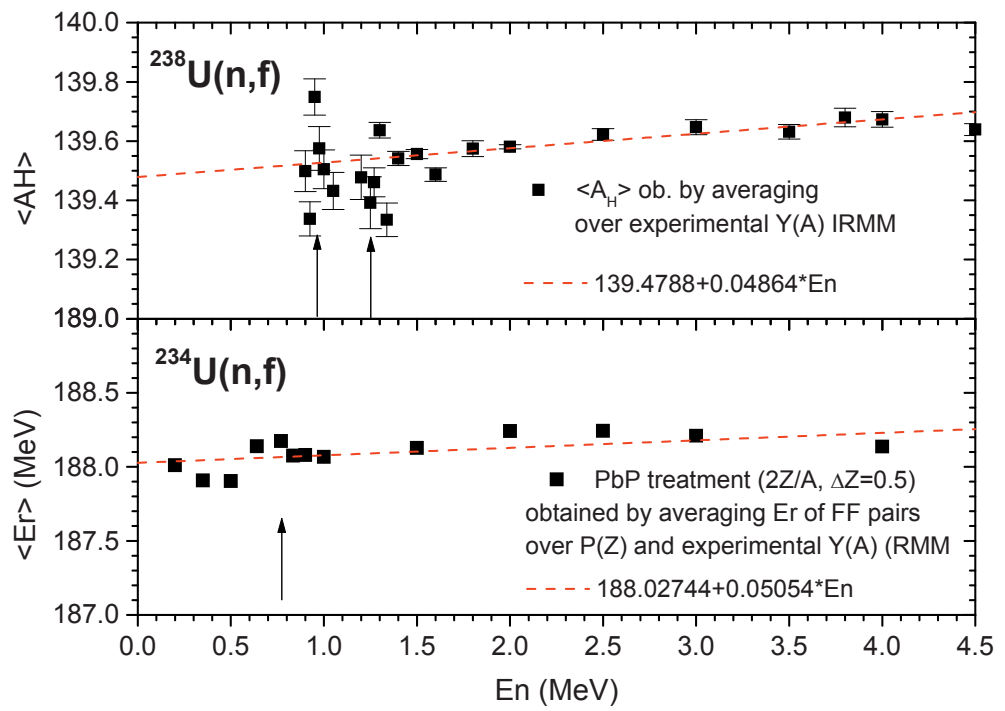

Fig.3. Upper part $<A_{H}>$ as a function of En for ${ }^{238} U(n, f)$, lower part $<E r>$ as a function of En for ${ }^{234} U(n, f)$.

Other average quantities characterizing the fragments, obtained by the Point-by-Point (PbP) treatment, such as the first neutron separation energy from $\mathrm{FF}\left\langle\mathrm{Sn}_{1}\right\rangle$, the level density parameter $\langle a\rangle$ and the total excitation energy at full acceleration $<\mathrm{TXE}>$ also exhibit visible fluctuations around the En of resonances, details are given in Ref.[4]. Fluctuations are visible in the prompt 
neutron multiplicity, too, see the PbP model results plotted with full red circles in the upper and lower parts of Fig.4.

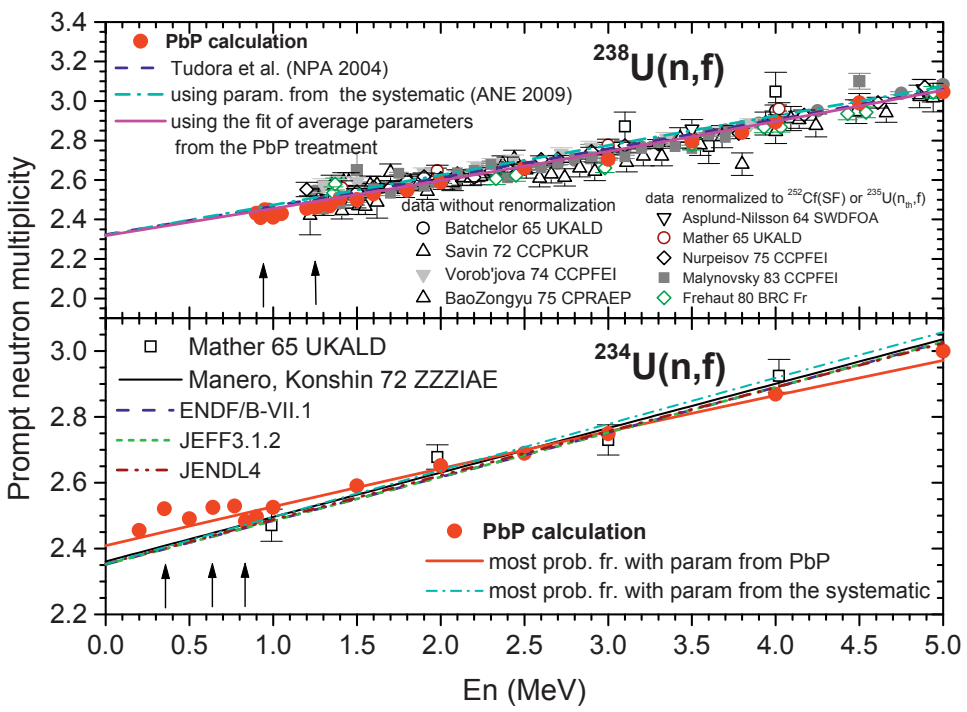

Fig.4. Total average prompt neutron multiplicity of ${ }^{238} U(n, f)$ (upper part) and ${ }^{234} U(n, f)$ lower part: $P b P$ results with full red circles and most probable fragmentation results using different average model parameters (as indicated in the figure legends) with different types of lines in comparison with experimental data (plotted with different symbols).

\section{Multi-modal fission cross-section calculation}

The statistical model with sub-barrier effects extended to include the multi-modal fission, contained in the STATIS code (for details see [4] and references therein), is used to provide the multi-modal fission cross-sections together with the cross-sections of competitive processes through the compound nucleus mechanism. The consistency of present neutron induced cross-section calculation is assured by the very good agreement of all cross-section results (for elastic, inelastic, capture and fission) with existing experimental data and recent released evaluations. For details see Ref.[4]. Details regarding the direct interaction mechanism treated by the coupled-channel method and the deformed optical model parameterisations used, the treatment of level densities at ground state and fission path deformations, the enhancement factors and the fission barrier parameters for each mode can be found in Ref.[4] (and references therein), too.

The vibrational resonaces in the fission cross-sections of ${ }^{238} \mathrm{U}$ (placed at around $0.95 \mathrm{MeV}$ and $1.25 \mathrm{MeV}$ ) and of ${ }^{234} \mathrm{U}(\mathrm{n}, \mathrm{f})$ (at around $0.35 \mathrm{MeV}, 0.6 \mathrm{MeV}$ and especially at $0.8 \mathrm{MeV}$ ) are well described by our results (see for instance the red lines in the upper parts of Fig.1 and 2).

The multi-modal fission concept also can give an explanation of the correlation between the subbarrier resonances of the fission cross-section and the pronounced variation of FF properties (for instance $<\mathrm{TKE}>$ ) at almost the same En values.

In the case of ${ }^{238} \mathrm{U}$, as it can be seen in the upper part of Fig.5, both calculated fission mode cross-sections (blue dashed line for S1 mode and green solid line for S2 mode) exhibit resonances at around $0.95 \mathrm{MeV}$ and $1.25 \mathrm{MeV}$, the resonant behaviour being a little bit more pronounced in the case of the S2 fission mode. The experimental fission mode weights (branching ratios) [2] plotted in the lower part of Fig.5 (with blue squares for S1 and green circles for S2) exhibit pronounced variations around the fission cross-section resonances with an increase of the S1 weight and a respective decrease of the S2 weight. As observation in the En range of interest for vibrational resonances the contribution of the symmetric super-long mode SL being very low was neglected. 
The average total kinetic energy of the $\mathrm{S} 1$ mode $\langle\mathrm{TKE}\rangle_{\mathrm{S} 1}$ has always the highest value because of the split in almost spherical fragments in connection with the closed shells $\mathrm{N}=82$ and $\mathrm{Z}=50$ and the lowest distance between their charge centres. Consequently the increase of $\langle\mathrm{TKE}\rangle_{\mathrm{S} 1}$ around the resonance energies (due to the increase of the $\mathrm{S} 1$ weight) determines the behaviour of the average $<\mathrm{TKE}>$.

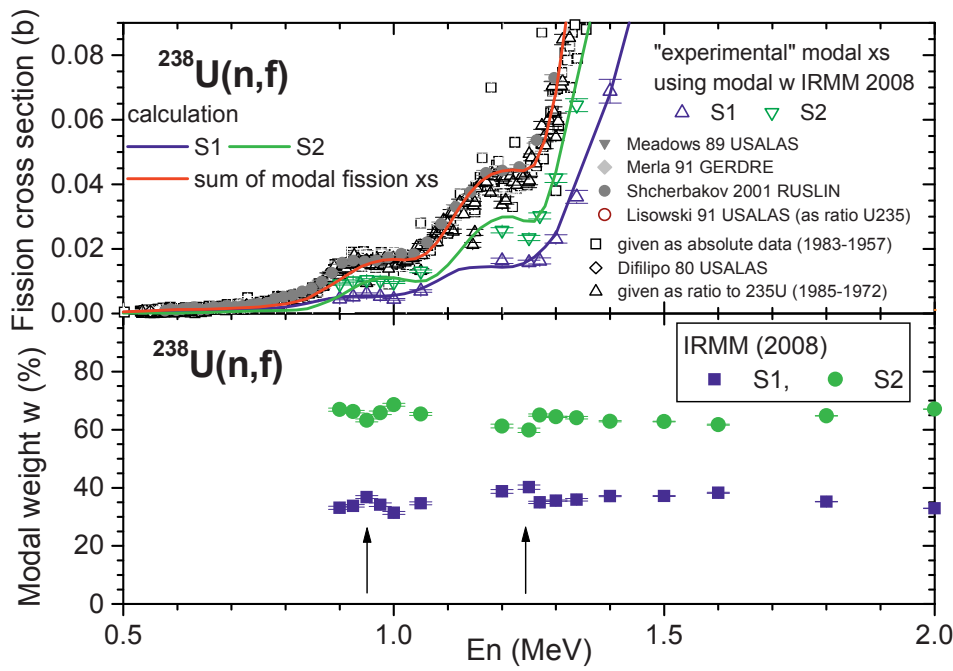

Fig.5. ${ }^{238} \mathrm{U}(\mathrm{n}, \mathrm{f})$ upper part: fission mode cross-sections (blue dashed line for $\mathrm{S} 1$ and green solid line for S2) and ther sum (red bulk line) in comparison with experimental data, the En range was chosen to focus the sub-barrier resonances. Lower part: experimental modal weights of S1 mode (blue squares) and S2 mode (green circles).

\section{Conclusions}

The correlation between the sub-barrier resonant behaviour of fission cross-section of fertile actinides (pre-scission stage) and the visible fluctuations of their fission fragment and prompt neutron data (post-scission stage) around the incident energies of resonances is outlined and supported by quantitative results in the case of two fissioning systems ${ }^{234,238} \mathrm{U}(\mathrm{n}, \mathrm{f})$.

Through the Point-by-Point treatment of prompt neutron emission and the multi-modal fission concept (also included in the statistical model with sub-barrier effects used in nuclear reaction calculations) we arrived at a quantitative explanation of the observed fluctuations.

New calculations of neutron-induced cross-sections of ${ }^{238,234} \mathrm{U}$ have been performed making use of recent coupled channel deformed optical model parameterisations and a refined model for the fission channel treatment. The consistency is proven by all integral and differential cross-sections obtained in good agreement with existing experimental data and recent evaluations.

The PbP model used together with experimental fragment distributions to provide all quantities characterizing the prompt neutron emission (as a function of fragment, of TKE and total average values) allow i) the quantitative support of the correlation between the sub-barrier resonances of the fission cross-section and the variation of fragment and prompt neutron properties and ii) to validate the predictions of previous calculations and systematics regarding the prompt neutron emission.

\section{References}

1. F. Vivès, F.-J. Hambsch, H. Bax, S.Oberstedt, Nucl. Phys.A 662 (2000) 63-92

2. E. Birgersson, A. Oberstedt, S. Oberstedt, F.-J. Hambsch, Nucl. Phys.A 817 (2009) 1-34

3. A. Al-Adili, F.-J. Hambsch, S. Oberstedt, S. Pomp, Sh. Zeynalov, Nucl. Instr. Methods A 624 (3) (2010) 684-690

4. A. Tudora, F.-J. Hambsch, S. Oberstedt, Nucl. Phys.A 890-891 (2012) 77-101 